\title{
O Sistema de Informação em Saúde como instrumento da política de recursos humanos: um mecanismo importante na detecção das necessidades da força de trabalho para o SUS
}

\author{
The H ealth Information System as a human \\ resources policy tool: an important mechanism \\ for the detection of labor force needs \\ in the U nified H ealth System
}

Kátia Rejane de M edeiros 1

Heleny de Oliveira Pena M achado 1

Paulette Caval cante de Albuquerque 1

Garibaldi Dantas Gurgel Junior 1

\footnotetext{
1 Observatório de Recursos Humanos, NESC/CPqAM/Fiocruz. Av. M oraes Rego $s / \mathrm{n}$, Cidade Universitária, Campus da UFPE, 50670-420, Recife PE. kmedeiros@cpqam.fiocruz.br
}

Abstract This article considers the potential of the $\mathrm{H}$ ealth Information System to aid decision making processes in H uman Resource $M$ anagement of the SUS [Brazilian U nified H ealth System]. The experience is described of the Pernambuco State Station, pertaining to the Human Resource O bservatory N etwork of OPAS [ Pan American $\mathrm{H}$ ealth Organization] with headquarters at the Collective H ealth Department of Aggeu M agalhães Research Center. The objective was to describe the development of new methodologies using $\mathrm{H}$ ealth Information Systems that can be used to pinpoint deficiencies in health services, improve the distribution of health workers and consequently optimize medical attendance.

Key words Information systems, Human resource, Access
Resumo Este artigo discute a potencialidade do uso dos Sistemas de Informação em Saúde, em apoio ao processo de tomada de decisão, na gestão da política de recursos humanos para o SUS. Descreve-se a experiência da Estação Pernambucana da Rede de O bservatórios de Recursos H umanos das Américas, da O rganização Pan-Americana de Saúde (OPAS), sediada no Departamento de Saúde Coletiva, do Centro de Pesquisas Aggeu $M$ agalhães, no desenvolvimento de metodologias para o manejo dos sistemas informacionais, com o objetivo de identificar áreas críticas na oferta de serviços de saúde e, conseqüentemente, carências de profissionais médicos. A metodologia apresentada pode ser utilizada como um instrumento de gestão para melhorar a distribuição de profissionais, de forma a atender às necessidades de saúde da população.

Palavras-chave Sistemas de informação, Recursos humanos, A cesso 


\section{Introdução}

$N$ as últimas décadas, um conjunto de atores institucionais e a sociedade civil organizada promoveram o que se chamou de Reforma da Reforma Sanitária Brasileira, um movimento social com o objetivo de construir o Sistema Ú nico de Saúde (Cordeiro, 2001). Dentre os cinco pontos centrais do redirecionamento do sistema, um aponta para a questão da gestão e do desenvolvimento dos recursos humanos (Brasil, 1986).

Para o setor saúde, a preocupação com os recursos humanos não é nova e pode- se dizer que é "crônica" a constatação de que esse é um nó crítico do sistema de saúde no país. N esse sentido, segundo Pierantoni (2001), o esforço para abordagem desta complexa problemática apresenta os recortes encontrados nas publicações deste campo do conhecimento que vão desde as abordagens da gestão dos recursos humanos até a sociologia das profissões do setor saúde.

Desde a criação do SUS é iminente a necessidade de se rever a gestão dos recursos humanos tanto no que se refere à sua distribuição geográfico-espacial, quanto às formas de gestão, capacitação de pessoal e regulação profissional. Zucchi (1998) e Santana (1996), afirmam que, no Brasil, os recursos humanos em saúde são caracterizados por graves insuficiências qualitativas e quantitativas, com uma distribuição desigual que impõe um envolvimento cada vez maior de gestores com esta questão.

No contexto atual de demandas crescentes para os gestores da saúde e, após mais de uma década da implementação do SUS, particularmente os gestores municipais vêm enfrentando com dificuldades variadas tais como a fixação de profissionais de saúde em municípios de pequeno porte; as imposições da Lei de Responsabilidade Fiscal; e a necessidade de adoção de novas tecnologias de gestão que o processo de Reforma Sanitária exige.

Essa temática reveste-se de especial importância dada a natureza das organizações de saúde fortemente dependentes de seus operadores, já que são classificadas como organizações profissionais (Dussault, 1992).

Este artigo visa refletir sobre as possibilidades de análise da distribuição de recursos humanos em diferentes serviços de saúde, nos municípios e Estados da federação, utilizandose de dados dos Sistemas de I nformações Assistenciais do SUS.
Assim, partindo-se dos dados dos Sistemas de Informação, busca-se uma relação entre possíveis vazios de recursos humanos e vazios assistenciais e, de modo contrário, correlaciona-se a boa dispersão assistencial com a boa distribuição de recursos humanos. Esse dimensionamento pode ser estimado a partir da caracterização da oferta/não- oferta, de serviços de saúde, tendo como pressuposto que a disponibilidade de profissionais de saúde está intrinsecamente ligada a esse processo.

\section{A potencialidade dos Sistemas Nacionais de Informações em Saúde}

Os Sistemas de Informação em Saúde, sejam eles assistenciais ou epidemiológicos, têm sido apontados como ferramentas importantes para o diagnóstico de situações de saúde com vistas a intervenções mais aproximadas do quadro de necessidades da população. É possível utilizar as bases de dados nacionais que, alimentadas regularmente por municípios e Estados, estão sendo ainda subutilizadas no seu potencial de instrumentalizar a tomada de decisões e de produção científica.

Esta afirmação é fundamentada tanto pelas características próprias dos sistemas informacionais que agregam milhões de dados e permitem inúmeras possibilidades de uso, quanto pela observação de que os gestores de sistemas de saúde têm demonstrado uma preocupação crescente em conhecer e apropriar-se do elenco destes recursos, o que possibilita definições mais acertadas na priorização de ações no setor saúde.

N ota-se também, entre as instituições de pesquisa, uma preocupação em agilizar e ampliar a função destes sistemas, mas, apesar dos esforços destinados em utilizar com maior freqüência tais instrumentos, verifica-se ainda pouca utilização das bases de dados, diante do potencial de variáveis e informações disponibilizadas.

Esses Sistemas de Informações têm merecido atenção na produção científica. Vale destacar que, nos estudos disponíveis, são identificadas algumas limitações dos Sistemas de Informações em Saúde como, por exemplo, o seu nível de desagregação e a falta de unicidade ou intercomunicação entre os sistemas já que, no Brasil, eles são de responsabilidade de distintas agências nacionais e de diversos setores do próprio M inistério da Saúde (Baldijão, 1992; Car- 
val ho, 1997). Estas limitações necessitam ser superadas.

Entretanto, ressal vadas as restrições, a capacidade dos Sistemas de I nformações Assistenciais é considerada elucidativa e com muitas outras possibilidades além daquelas relacionadas à remuneração da produção de serviços e vigilância epidemiológica.

Além do montante de variáveis disponíveis, o sistema SI H /SU S apresenta os seguintes aspectos positivos: identificação do paciente; caracterização do hospital e gestor (número de leitos, clínicas disponíveis, meios diagnósticos e terapêuticos), entre outros (Levcovitz, 1993); sua agilidade (disponíveis na internet em até 60 dias) e a característica de ser a única fonte regular e universal sobre morbidade no país (Almeida,1996; Baldijão, 1992; Buss, 1993). Estas características fazem deste sistema uma importante ferramenta para avaliação e criação de indicadores de desempenho da rede assistencial e de avaliação de sistemas de saúde, identificando gastos, oferta de serviços, demanda e cobertura assistencial.

O SIA/SUS também apresenta vantagens para o estudo da oferta de serviços, engl obando todos os procedimentos de assistência à saúde que não exijam internação, sendo amplamente descentral izado nos Estados e municípios, além de refletir a produção mensal das unidades do SUS. N este sistema, também estão disponíveis as Fichas de Programação FísicoOrçamentária (FPO) e as Fichas de Cadastro das U nidades (FCA) que, mesmo não sendo atualizadas com a periodicidade necessária, permitem a caracterização das unidades e dos gestores. Estas informações garantem ao SIA/ SU S condições e capacidade de disponibilizar elementos referentes ao desempenho dos gestores na seleção e implementação de prioridades assistenciais (Carvalho, 1997).

O SIA/SUS permite a caracterização da rede, num quadro geral de disponibilidade de recursos tecnológicos e humanos por município, mesorregião municipal, Estado ou região.

Recentemente foram apontadas potencialidades inovadoras dos Sistemas de Informações Assistenciais, especialmente o SIH/SUS, como fonte complementar na vigilância e monitoramento de Doenças de Notificação Compulsória, representando um potencial auxiliar deste sistema em relação aos sistemas de informações epidemiológicas (M endes et al., 2000).

Os bancos de dados dos sistemas de informações são ferramentas fundamentais para ou- tros trabalhos, pois são bancos de dados que dispõem de informações de 12 milhões de internações e 1,3 trilhão de procedimentos ambulatoriais, num país que carece de informações. Vale ressaltar que são os municípios os responsáveis pela alimentação regular destes bancos de dados.

No entanto, estas informações são pouco exploradas no que se refere à área de recursos humanos em saúde, com enfoque na necessidade de reorganização da assistência. Vale ressaltar, neste sentido, que o potencial dos sistemas de informações em saúde, como demonstradores de acesso e oferta de serviços de saúde para a população, pode ser amplamente utilizado.

O estudo de Buss (1993), partindo do Sistema de Informações H ospitalares (SIH/SUS), e explorando variáveis da assistência hospitalar, demonstrou consideráveis disparidades na oferta de serviços de saúde no país no período de 1984 a 1991. N este estudo chama a aten ção o predomínio da oferta de serviços de saúde naquelas regiões consideradas mais ricas representando uma desigual dade na oferta eno acesso aos serviços de saúde pelas diferentes populações e estratos sociais.

A crescente responsabilização dos municípios na execução da política de saúde aponta para um crescimento significativo dos sistemas de saúde locais, como demonstraram Scatena e Tanaka (1998) ao analisarem a distribuição dos estabelecimentos de saúde no Brasil no período de 1984 a 1992. Os autores observaram que, desde 1985, os municípios já vinham incrementando suas redes de estabelecimentos de saúde, embora ocorra em 1990 um crescimento mais acelerado quando, simultaneamente, vão se reduzindo as participações dos Estados e da União nesta oferta.

Estes dados sugerem a possibilidade de investigação e utilização dos sistemas de informação no processo de gestão de recursos humanos em saúde que estão na base do processo de trabal ho responsáveis por esta produção.

Tomando a categoria médica como central nas organizações de saúde e analisando a história da formação das escolas médicas, vê-se que estas contribuem para a distribuição não programada desses profissionais, já que a formação dessas escolas, no Brasil, atende a conjunturas e interesses políticos, econômicos ou técnico-científicos (Dal Poz \& Varella, 1995), o que faz com que a distribuição destes profissionais, seja apontada como um elemento crítico 
na gestão de recursos humanos, para o sistema de saúde no Brasil.

\section{Metodologia desenvolvida}

0 estudo utilizou as fontes de informações secundárias disponíveis nos Sistemas de Informações Assistenciais (Sistema de Informações Ambulatoriais - SI A/SUS e Sistemas de Informações Hospitalares - SIH/SUS), no ano de 1999.

N os dois sistemas, selecionaram-se informações cadastrais de unidades e de produção de serviços, o que permitiu a construção de variáveis e indicadores.

Para a construção de indicadores, quais sejam: consulta/habitante/ano e internação/habitante/ano, recorreu-se ao Tabnet/D atasus, que dispõe de estimativas do IBGE de população para o ano de 1999. Antes da construção do indicador, foi necessário fazer algumas pesquisas e seleções no banco de dados, estabelecendo-se estratégias comuns para sua exploração, haja vista que, a partir de novembro de 1999, a tabela do SIA/SU S passou por mudanças devido à criação de 12 novos grupos de procedimentos e à implantação de classificação de oito dígitos para seus procedimentos. As seleções realizadas no banco de dados do SIA/SU S para a produção deste estudo restringem-se, apenas, a procedimentos médicos.

$\mathrm{N}$ a ausência de critérios prescritivos, para adoção de estratificação populacional, e pelo registro de variadas opções de estratificação, utilizadas em estudos sobre a realidade de saúde, no nível municipal (Heimann, 1992; Gershman, 1995; Carvalho, 1995 e Brasil, 1995), a pesquisa identificou faixas que se mostraram com maior poder discriminatório para a especificidade do objeto de estudo na realidade brasileira.

Assim, a pesquisa de população pelo Tabnet, permitiu a criação de 11 estratificações municipais por faixas de população. Essas faixas foram cruzadas com os indicadores criados no SIA/SUS e SIH/SUS, possibilitando uma correlação entre o perfil populacional dos municípios e a oferta/acesso a serviços de saúde. Através das informações cadastrais do SAI/SUS identificou-se o perfil assistencial das redes de saúde em municípios, Estados e regiões do país. Portanto, a partir desta etapa da investigação, verificou-se a distribuição dos equipamentos de saúde.
Para verificar a produção ambulatorial, 0 indicador consulta/habitante/ano foi estratificado e então cruzado com faixas populacionais, o que permitiu evidenciar os municípios e Estados mais críticos com relação à oferta de consulta médica diante dos parâmetros tradicionalmente preconizados pelo M inistério da Saúde.

$\mathrm{N}$ o SIH/SU S pesquisou-se somente dados de produção de internações, os quais foram selecionados segundo município de residência e ocorrência, no ano de 1999, o que favoreceu a identificação de municípios pólos, para atenção médica hospitalar, e municípios onde inexiste unidade hospitalar. 0 cruzamento do indicador internação/habitante/ano e as faixas populacionais permite a identificação da presença de oferta de atenção médica hospitalar, segundo o porte do município.

Com a agregação dos três indicadores acima descritos, foi criado um indicador complexo, que permitiu a construção de uma tipologia segundo "situação geral de acesso a serviços de saúde" (ambulatoriais e hospitalares) dos municípios, estabelecendo quatro faixas de nível de acesso aos serviços, já que elas se mostraram sensíveis para discriminar as diferentes situações da realidade do país.

Para análise da "situação geral de acesso", considerou-se a distribuição de freqüência em torno dos valores observados situados, abaixo, iguais e acima dos parâmetros assistenciais do M inistério da Saúde no ano de 1981. Apesar das controvérsias acerca da desatualização dos parâmetros de 1981, definiu-se pela sua utilização, visto ser o que se encontra em vigor, ou seja, trabalhou-se com o referencial 2 consulta/habitante/ano, e 100 internação/1.000habitantes/ano. Sabe-se do processo de revisão desses parâmetros, no âmbito do Conselho N acional de Saúde.

A primeira faixa corresponde à situação na qual o nível de acesso predominante no Estado está muito abaixo, dos padrões recomendados pelo M inistério da Saúde, isto é, Estados nos quais, no mínimo $50 \%$ dos municípios apresentam indicadores abaixo da metade do recomendado.

A segunda faixa corresponde à situação, na qual o nível de acesso predominante no Estado está em um nível intermediário, isto é, embora esteja abaixo dos padrões recomendados pelo M inistério da Saúde, estes Estados têm, no mínimo, 50\% dos municípios cuja distribuição dos indicadores está acima da metade do recomendado. 
A terceira faixa corresponde à situação, na qual o nível de acesso predominante no Estado está adequado em relação aos padrões recomendados pelo M inistério da Saúde.

Por fim, a quarta faixa corresponde à situação na qual a oferta apresentada se encontra acima dos referidos parâmetros.

Os dados gerais das tabelas foram processados com recursos do programa Epi-info versão $6.04 \mathrm{~b}$, o que permitiu a construção de tabulações segundo faixas/estratos.

\section{Resultados}

Os resultados apresentados no quadro 1 refletem a situação dos Estados por níveis de oferta, considerando os três indicadores trabalhados: consulta/habitante/ano; e internação/habitante/ano, por município de ocorrência e internação/habitante/ano por município de residência. Destaca-se que o estudo se referencia a quatro estratos definidos, a saber:

- o primeiro corresponde ao nível de acesso abaixo da metade do recomendado pelo M inistério da Saúde;

- o segundo corresponde ao nível de acesso cuja distribuiçãao dos indicadores está acima da metade do recomendado, sem atingir os parâmetros;

- o terceiro corresponde ao nível considerado adequado em relação aos padrões do M inistério da Saúde; e

- o quarto nível corresponde à situação na qual a oferta se encontra acima, dos referidos parâmetros.

Os níveis de 1 a 4 correspondem, respectivamente, às situações de acesso caracterizadas como: crítica, intermediária, razoável eboa, sugerindo um quadro de recursos humanos (profissional médico) equivalente.

Este quadro permite uma dupla análise, pois é possível considerar cada indicador isoladamente, por Estado, e o indicador complexo, também tomado por Estado.

Em relação ao indicador consulta/hab./ano, os resultados expressam diferenças regionais importantes.

Os Estados do N orte e N ordeste apresentam indicadores concentrados nas faixas 1 e 2 , ou seja, com níveis "críticos" e "intermediários", de acesso a consultas médicas. Na Região Norte, apesar do predomínio de Estados no nível 1, destacam-se Tocantins e Rondônia, por se posicionarem na situação intermediária. No entanto, no Nordeste, observa-se o inverso, prevalece o nível intermediário, mas os Estados da Bahia, M aranhão e Piauí refletem situações críticas nestes indicadores.

A Região Sudeste destaca-se do conjunto do país ao apresentar os níveis 3 e 4, sinalizando padrões "razoável" e "bom", de acesso à consulta médica, sendo que o Estado com nível bom restringe-se ao Rio de Janeiro.

As Regiões Sul e Centro-Oeste apresentam padrões de acesso à consulta médica semel hantes, oscilando entre "intermediário" e "razoável". Destacam-se, para o último nível, os Estados do Paraná e Goiás.

0 cruzamento do indicador consulta médica, por habitante/ano, segundo Estado da federação, permite observar que os Estados nos quais os resultados apontam ausência ou precariedade de acesso, existe escassez de profissional médico, portanto, no Acre, Amazonas, Amapá, Roraima, Bahia, M aranhão e Sergipe, os indicadores sugerem esta situação, ou seja, torna-se necessária a adoção de estratégias voltadas para incorporação e fixação desses profissionais, em 582 municípios de tais Estados.

Quanto à oferta de internação, por município de residência, a situação é heterogênea. No N orte, prevalece a situação crítica, exceto para Rondônia, que se destaca por apresentar uma "boa" situação de acesso (faixa 4) neste indicador.

O N ordeste reflete uma melhora, pois apresenta certa proporcionalidade entre as faixas "intermediária" e "razoável".

o Sudeste e o Sul apresentam o mesmo nível para todos os Estados, representados pela faixa "razoável" quanto ao acesso à internação por residência.

No Centro-O este, a situação é heterogênea, oscilando entre os níveis "intermediário" e "bom", caso do M ato Grosso do Sul.

Vale salientar que os únicos Estados brasileiros a apresentarem uma situação boa, quanto a este indicador, são os Estados do Tocantins eAmazonas.

Quanto à oferta de internação, por município de ocorrência, o N orte, apresenta uma predominância do nível "crítico", excetuando-se os Estados de Rondônia, com nível "razoável" e o Amazonas com nível "intermediário".

O Nordeste oscila entre os níveis "crítico" e "razoável". N este último estão Rio Grande do Norte, Ceará e Bahia. N ota-se a predominância do nível "crítico". 
Quadro 1

Síntese dos indicadores de acesso a serviços médicos, Brasil, 1999.

\begin{tabular}{|c|c|c|c|c|}
\hline Regiões & Consulta & Int. Res. & Int. Ocor. & Acesso \\
\hline \multicolumn{5}{|l|}{ Norte } \\
\hline \multicolumn{5}{|l|}{$A C$} \\
\hline \multicolumn{5}{|l|}{$A M$} \\
\hline \multicolumn{5}{|l|}{$A P$} \\
\hline \multicolumn{5}{|l|}{ PA } \\
\hline \multicolumn{5}{|l|}{ RO } \\
\hline \multicolumn{5}{|l|}{$\mathrm{RR}$} \\
\hline \multicolumn{5}{|l|}{ TO } \\
\hline \multicolumn{5}{|l|}{ Nordeste } \\
\hline \multicolumn{5}{|l|}{$A L$} \\
\hline \multicolumn{5}{|l|}{ BA } \\
\hline \multicolumn{5}{|l|}{$\mathrm{CE}$} \\
\hline \multicolumn{5}{|l|}{ MA } \\
\hline \multicolumn{5}{|l|}{ PB } \\
\hline \multicolumn{5}{|l|}{$P E$} \\
\hline \multicolumn{5}{|l|}{$\mathrm{PI}$} \\
\hline \multicolumn{5}{|l|}{ RN } \\
\hline $\mathrm{SE}$ & & & & \\
\hline
\end{tabular}

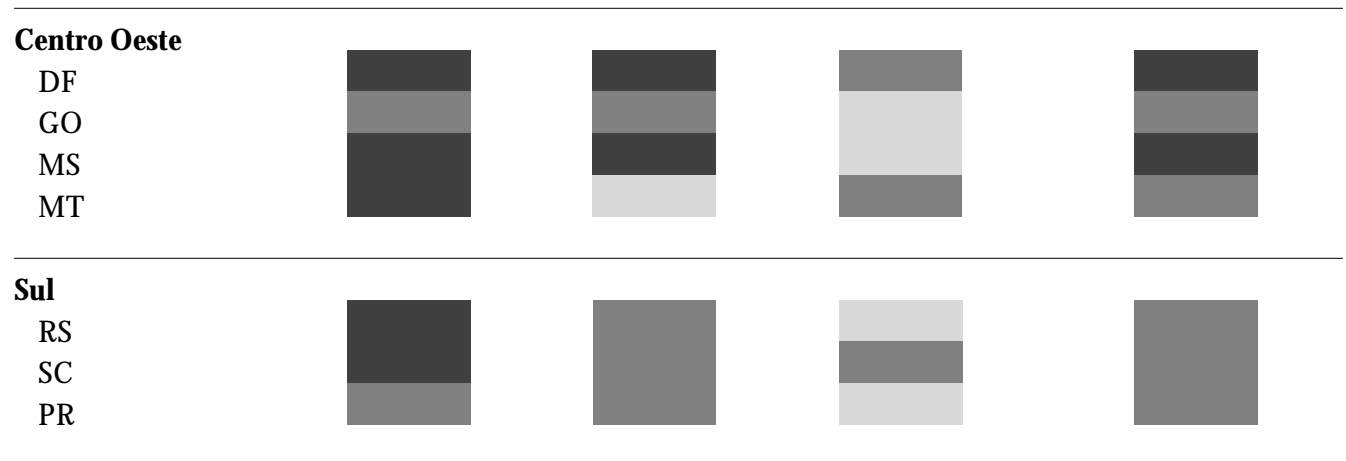

Sudeste
ES
M G
RJ
SP

Fonte: IBGE, SIH e SIA/SUS

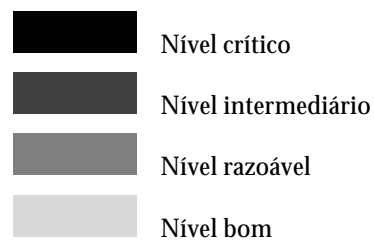


No Sudeste, observam-se variações entre nível "crítico", para São Paulo e M inas Gerais, passando para "razoável", no Espírito Santo, preservando no Rio de Janeiro uma situação de acesso "boa".

As Regiões Sul e Centro-Oeste apresentam situações semel hantes, destacando-se com os melhores níveis, entre "razoável" e "bom".

Analisando-se, sob o ponto de vista do indicador complexo, confirma-se o quadro das desigualdades regionais de acesso a serviços de saúde e, conseqüentemente, sugere-se fortemente, uma má distribuição de profissionais médicos no país.

Há uma predominância dos piores indicadores na região $\mathrm{N}$ orte, seguida pela região $\mathrm{N}$ ordeste, embora se destaquem, nas duas regiões, respectivamente: Rondônia e Rio Grande do N orte e Ceará.

A Região Sudestemantém a situação de meIhor desempenho, sendo que o Rio de Janeiro continua, destacadamente, apresentando o meIhor nível de acesso da região e do país. Os demais Estados apresentam desempenho na faixa definida dentro dos parâmetros do M inistério da Saúde.

A Região Sul apresenta-se bastante homogênea, de modo que todos os Estados encontram-se no mesmo nível, isto é, todos também na faixa definida dentro dos parâmetros do M inistério da Saúde.

O Centro-O este apresenta proporcionalidade entre as faixas "intermediária", no caso do Distrito Federal e M ato Grosso, e a faixa "razoável" em Goiás e M ato Grosso do Sul.

Com base nestes resultados, compôs-se 0 quadro 1 com uma distribuição das regiões, segundo a situação geral de acesso a serviços de saúde, de modo a destacar a gradação dos níveis crítico, intermediário, razoável e bom para cada uma das regiões brasileiras.

\section{Conclusões}

A experiência confirma a possibilidade de utilização mais intensa do volume de informações disponíveis nos Sistemas de I nformações Assistenciais, além de seu potencial para subsidiar 0 incremento das Políticas de Recursos H umanos em Saúde. A construção de uma metodologia adequada permite a transposição da informação assistencial à necessidade presumida de profissionais de saúde.

N este ponto, reside outro potencial dos Sistemas de Informações Assistenciais, resultante do processo de desenvolvimento de métodos capazes de se apropriar, adequadamente, dos dados e transformá-los em informação estratégica para a gestão através da metodologia desenvolvida e do banco de dados criados pela pesquisa.

Utilizando-se dos sistemas de informações, é possível aferir a necessidade presumida, quantitativa/qualitativa de recursos humanos em saúde, e outras informações necessárias para a gestão do sistema, tornando relevantes as seleções realizadas no banco de dados do SI A/SUS, que dizem respeito a procedimentos médicos que possi bilitam a localização de vazios assistenciais, ou seja, onde há carência destes profissionais, bem como de especialidades médicas.

Através da metodologia e do banco de dados criados, há a possibilidade de explorar, com segurança, determinada categoria profissional e identificar, por exemplo:

- a produção assistencial e a existência presumida de profissionais médicos em municípiospólo, para atenção médica ambulatorial e hospitalar no país, Estados e mesorregiões;

- a produção assistencial e a existência presumida de profissionais médicos dos municípios, do Programa de Interiorização dos Trabalhadores de Saúde, do M inistério da Saúde;

- a produção assistencial e a existência presumida de profissionais médicos dos municípios brasileiros em condição crítica de acesso, aos serviços de saúde, por Estado da federação.

Portanto, torna-se oportuno o desenvolvimento de metodologias que investiguem outras bases de dados, com vistas ao conhecimento da real necessidade desses profissionais, para meIhor entender o problema da carência de médicos no país. 


\section{Colaboradores}

$K$ M edeiros trabalhou na revisão bibliográfica e elaboração do texto; P Caval canti eH M achado, na definição do plano e análise dos dados coletados na pesquisa; e G Gurgel, na revisão final do texto para apresentação à revista.

\section{Referências bibliográficas}

Almeida M F 1996. Algumas considerações sobre os sistemas de informação em saúde da França e do Brasil. Informe Epidemiológico do SUS 5(4):7-12.

Baldijão M FA 1992. Sistemas de informação em saúde. Revista São Paulo em Perspectiva 6(4):21-28

Brasil. M inistério da Saúde 1986. Relatório da 8ạ Conferência N acional de Saúde. M inistério da Saúde, Brasília.

Brasil. M inistério da Saúde 1995. Avaliação do funcionamento dos Consel hos Estaduais e M unicipais de Saúde. M inistério da Saúde, Brasília.

Buss PM 1993. Assistência hospitalar no Brasil (19841991): análise preliminar baseada no Sistema de Informação H ospitalar do SUS. Informe Epidemiológico do SUS 2(2):4-42.

Carvalho AI 1995. Consel hos de Saúde no Brasil: participação cidadã e controle social. IBAM , Rio de Janeiro.

Carvalho DM 1997. Grandes sistemas nacionais de informação em saúde: revisão e discussão atual. Informe Epidemiológico do SU S 6(4):7-46.

Cordeiro H 2001. Descentralização, universalidade e equidade nas reformas da saúde. Ciência e Saúde Coletiva 6(2):319-328.

Dal Poz M \& Varella C 1995. Estudo de condições de formação e exercício profissional em saúde no Brasil, pp. 75-106. In Organização Pan-Americana de Saúde. Recursos H umanos em Saúde no M ercosul. Fiocruz, Rio de Janeiro.

Dussault G 1992. A gestão dos serviços públicos de saúde: características e exigências. Revista de Administração Pública 26(2):8-19.
Gershman S 1995. A democracia inconclusa. Fiocruz, Rio de Janeiro.

H eimann L et al.1992. 0 município ea saúde. Hucitec, São Paulo.

Levcovitz E \& Pereira TRC 1993. SIH/SUS (Sistema AIH): uma análise do sistema público de remuneração de internações hospitalares no Brasil 1983-1991. UERJ/IM S, Rio de Janeiro.

M endes ACG et al. 2000. Avaliação do sistema de informações hospitalares SIH /SU S como fonte complementar na vigilância e monitoramento de doenças de notificação compulsória. Informe Epidemiológico do SUS 9(2):67-86.

Pierantoni CR 2001. As reformas do Estado, da saúde erecursos humanos: limites e possibilidades. Ciência e Saúde Coletiva 6(2):341-356.

Santana JP 1996. Recursos humanos: desafios para os gestores do SUS. Divulgação em Saúde (14):33-36.

Scatena JH G \& Tanaka OY 1998. Distribuição dos estabelecimentos de saúde no Brasil: para qual modelo caminhamos? Revista de Administração Pública 32(4): 7-25.

Zucchi P 1998. Funcionários por leito: estudos em alguns hospitais públicos e privados. Revista de Administração Pública 32(3):65-76.

Artigo apresentado em 11/12/2004

Aprovado em 12/04/2004

Versão final apresentada em 26/01/2005 